\title{
LV. Further researches in the undulatory theory of light
}

\author{
John Tovey Esq.
}

To cite this article: John Tovey Esq. (1836) LV. Further researches in the undulatory theory of light , Philosophical Magazine Series 3, 8:47, 270-272, DOI: 10.1080/14786443608648871

To link to this article: http://dx.doi.org/10.1080/14786443608648871

曲 Published online: 01 Jun 2009.

Submit your article to this journal $₫$

Џ Article views: 2

Q View related articles $\square$ 
acid acted on chromate of lead and took up some lead, particularly when the acid was for several days in contact with it: according to Mans, (Poggendorff's Annalen, band ix. p. 127.) it is not soluble in acetic acid.

Royal German Spa, Brighton,

Gustavus Schweitzer.

November 29, 1835.

LV. Further Researches in the Undulatory Theory of Light. By John Tover, Esq.

To the Editors of the Philosophical Magazine and Journal. Genthemen,

BSERVING that my paper on the relation between the length and velocity of a wave of light, inserted in your Number for January last, has received, in your Number for February, a favourable notice from your eminently scientific correspondent Professor Powell, I venture to send you a continuation of my researches.

My object now is to transform the general equations (3.) of that paper, into others adapted to any case of undulation in which the directions of the coordinates can be so taken that the displacements $\xi, \eta, \zeta$, may be regarded as functions of $x$ and $t$.

On the condition just stated, we have, by Taylor's formula,

$\Delta \xi=\frac{d \xi}{d x} \Delta x+\frac{d^{2} \xi}{d x^{2}} \cdot \frac{\Delta x^{2}}{2}+\frac{d^{3} \xi}{d x^{3}} \cdot \frac{\Delta x^{3}}{2.3}+\frac{d^{4} \xi}{d x^{4}} \cdot \frac{\Delta x^{4}}{2.3 .4}+\& \mathrm{c}$.

$\Delta \eta=\frac{d \eta}{d x} \Delta x+\frac{d^{2} \eta}{d x^{8}} \cdot \frac{\Delta x^{2}}{2}+\frac{d^{3} \eta}{d x^{3}} \cdot \frac{\Delta x^{3}}{2.3}+\frac{d^{4} \eta}{d x^{4}} \cdot \frac{\Delta x^{4}}{2.3 .4}+\& \mathrm{c}$.

$\Delta \zeta=\frac{d \zeta}{d x} \Delta x+\frac{d^{2} \zeta}{d x^{2}} \cdot \begin{gathered}\Delta x^{2} \\ 2\end{gathered}+\frac{d^{3} \zeta}{d x^{3}} \cdot \frac{\Delta x^{3}}{2.3}+\frac{d^{4} \zeta}{d x^{4}} \cdot \frac{\Delta x^{4}}{2.3 .4}+8 \mathrm{c}$.

Now, suppose the arrangement of the molecules in the state of equilibrium to be such that for every molecule on one side of $m$, within the sphere of its influence, there is another at an equal distance on the opposite side; then, if we substitute these expressions for $\Delta \xi, \Delta \eta, \Delta \xi$, in the first of the equations (3.), the sums $\Sigma . \phi(r) \Delta x, \Sigma . \varphi(r) \Delta x^{3}, \Sigma \cdot \psi(r) \Delta x^{3}$, $\Sigma . \psi(r) \Delta x^{5}, \quad \Sigma . \psi(r) \Delta y \Delta x^{2}, \quad \Sigma . \psi(r) \Delta y \Delta x^{4}$, $\Sigma . \psi(r) \Delta z \Delta x^{2}, \quad \Sigma . \psi(r) \Delta z \Delta x^{4}, \& c$., in which the degrees of the products of the variations are odd, will vanish; because, whatever be the signs of $\Delta x, \Delta y, \Delta z$ for any molecule, these signs will all be changed for the corresponding mole- 
cule on the opposite side of $m$; consequently the signs of the odd products of the variations will be changed, while the absolute values of the variations and of their products remain the same for both molecules. This supposition respecting the arrangement of the molecules is due to $M$. Cauchy, and appears very probable; because it seems impossible to conceive how the equilibrium could subsist unless it were true. It is also probable that the sphere of the influence of each molecule comprehends a great number of other molecules; and accordingly we shall assume this as an hypothesis. Now, as we cannot suppose the molecules, in their state of equilibrium, to be more crowded in one part of the sphere than another, it follows that the terms of the other sums $\Sigma . \psi(r) \Delta y \Delta x^{3}$, $\Sigma . \psi(r) \Delta y \Delta x^{5}, \Sigma . \psi(r) \Delta z \Delta x^{3}, \Sigma . \psi(r) \Delta z \Delta x^{5}, \& c$., in which there are odd powers of the variations, will be about half of them positive and half negative, and will nearly destroy each other, and consequently these sums will nearly vanish. Neglecting them as well as the former, the first of the equations (3.) becomes, by the substitution,

$$
\frac{d^{2} \xi}{d t^{2}}=m \Sigma \cdot\left\{\left(\phi(r)+\psi(r) \Delta x^{2}\right) \cdot\left(\frac{d^{2} \xi}{d x^{2}} \cdot \frac{\Delta x^{2}}{2}+\frac{d^{4} \xi}{d x^{4}} \cdot \frac{\Delta x^{4}}{2.3 .4}+\& c^{2}\right)\right\}
$$

The second and third of the equations (3.) are of the same form as the first; consequently, if we transform them in the same manner, and, for the sake of abridgement, put

$$
\begin{aligned}
& \frac{m}{2} \Sigma \cdot\left(\phi(r)+\psi(r) \Delta x^{2}\right) \Delta x^{2}=s^{2} \\
& \frac{m}{2.3 .4} \Sigma \cdot\left(\Phi(r)+\psi(r) \Delta x^{2}\right) \Delta x^{4}=s^{\prime 2} \\
& \text { \&c. } \\
& \text { \&c. } \\
& \frac{m}{2} \Sigma \cdot\left(\phi(r)+\psi(r) \Delta y^{2}\right) \Delta x^{2}=s_{l}^{2} \\
& \frac{m}{2.3 .4} \Sigma \cdot\left(\phi(r)+\psi(r) \Delta y^{2}\right) \Delta x^{4}=s_{i}^{\prime 2} \\
& \text { \&c. } \\
& \text { \&c. } \\
& \begin{array}{l}
\frac{m}{2} \Sigma \cdot\left(\phi(r)+\psi(r) \Delta z^{2}\right) \Delta x^{2}=s_{\| \prime}{ }^{2} \\
\frac{m}{2.3 .4} \Sigma \cdot\left(\phi(r)+\psi(r) \Delta z^{2}\right) \Delta x^{4}=s_{\mu \prime}{ }^{2}
\end{array}
\end{aligned}
$$

\&c.

we shall have

\&c. 
272 Mr. Hopkins's Abstract of his Memoir on PhysicalGeology.

$$
\begin{aligned}
& \frac{d^{2} \xi}{d t^{2}}=s^{2} \cdot \frac{d^{2} \xi}{d x^{2}}+s^{\prime 2} \cdot \frac{d^{4} \xi}{d x^{4}}+\& \mathrm{c} . \\
& \frac{d^{2} \eta}{d t^{2}}=s_{1}^{2} \cdot \frac{d^{2} \eta}{d x^{2}}+s_{1}^{\prime 2} \frac{d^{4} \eta}{d x^{4}}+\& c . \\
& \frac{d^{2} \zeta}{d t^{2}}=s_{\| 1}{ }^{2} \cdot \frac{d^{2} \zeta}{d x^{2}}+s_{\| 1}{ }^{12} \frac{d^{4} \zeta}{d x^{4}}+\& \mathrm{c} .
\end{aligned}
$$

These equations show that the displacements in the three rectangular directions are, to the extent to which we have carried the approximation, independent of each other.

We have supposed the masses of the molecules to be all equal; but if the medium be composed of two fluids uniformly mixed, and if the masses of the molecules of one fluid be all equal to $m$, and of the other all equal to $m^{\prime}$, the equations (2.), which we have just obtained, will still be of the same form; because each of the sums $\Sigma$ may then be divided into two parts, one of which parts, multiplied by $m$, will embrace the molecules of one of the fluids, while the other part multiplied by $m^{\prime}$, will comprehend the molecules of the other fluid ; and the molecules of each fluid may be conceived to be arranged in the manner which we have supposed. In this way the equations may be extended to the case of any compound medium in which the elementary media are uniformly mingled.

In another communication 1 propose to deduce the integrals of the equations (2.), and point out the extent of their application. I am, Gentlemen, yours, \&c.

Evesham, Feb. 9, 1836.

John Tovey.

P.S. There are three typographical errors in my last paper. At page 9 , line 12 , for $x$ read $\Delta x$; page 10 , line 3 , for $d z^{4}$ read $\Delta z^{4}$; and line 22 , same page, for $s^{\prime 2} \operatorname{read} \frac{s^{12}}{3.4}$.

LVI. An Abstract of a Memoir on Physical Geology; with a further Exposition of certain Points connected with the Subject. By W. Hopkins, Esq., M.A., F.G.S., of St. Peter's College, Cambridge.

[Continued from p. 236.]

II. HAVING now reduced the determination of the horizontal directions of the fissures produced in the elevated mass to that of the fissure which would be produced in a plane lamina every point of which is subjected to known tensions, we may proceed with this latter problem. Our first object is to determine the direction in which the tensions have 